FORMATION Formation emploi

Revue française de sciences sociales

107 | juillet-septembre 2009

La formation professionnelle en Amérique latine

\title{
Lettre à un jeune chercheur
}

Présentation de l'ouvrage Pour une sociologie des rapports sociaux.

L'enseignement de Michel Arliaud

Sylvie Monchatre

\section{(2) OpenEdition}

1 Journals

Édition électronique

URL : http://journals.openedition.org/formationemploi/2036

DOI : 10.4000/formationemploi.2036

ISSN : 2107-0946

Éditeur

La Documentation française

Édition imprimée

Date de publication : 1 septembre 2009

Pagination : 93-97

ISSN : 0759-6340

Référence électronique

Sylvie Monchatre, «Lettre à un jeune chercheur », Formation emploi [En ligne], 107 | juillet-septembre

2009, mis en ligne le 14 décembre 2009, consulté le 30 octobre 2020. URL : http://

journals.openedition.org/formationemploi/2036 ; DOI : https://doi.org/10.4000/formationemploi.2036

(c) Tous droits réservés 


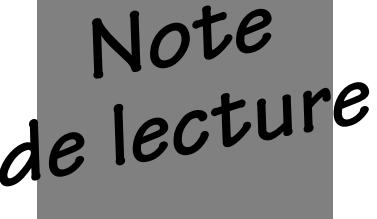

\section{Lettre à un jeune chercheur : Présentation de l'ouvrage "Pour une sociologie des rapports sociaux. L'enseignement de Michel Arliaud»}

Professionnaliser les jeunes chercheurs ne saurait vouloir dire renoncer à toute ambition théorique. La transmission d'expérience en ce domaine est d'autant plus précieuse qu'elle n'est guère fréquente. Cet ouvrage nous invite précisément

à un regard réflexif de la discipline sur elle-même, mais également sur les questionnements qu'elle soulève en pratique, ainsi que sur les paris interprétatifs qu'elle suppose. Et le tout prend la forme d'un témoignage vivant et enlevé...

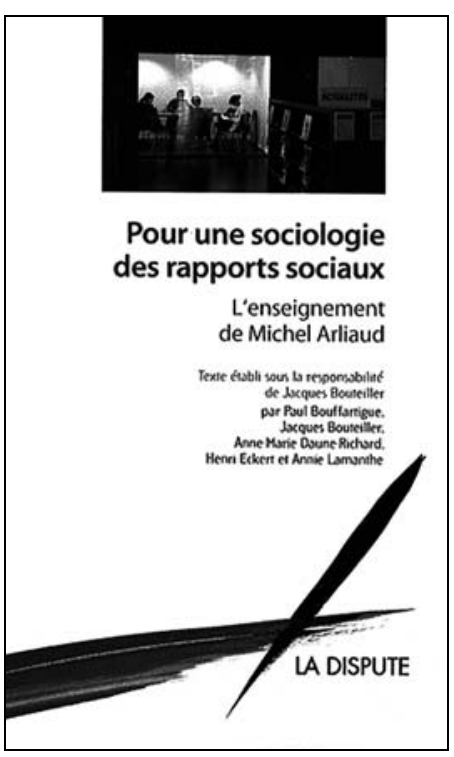

Par Sylvie Monchatre*

Heureux les futurs chercheurs, sociologues, enseignants et tous ceux qui sont curieux de la science du social! Cet ouvrage ne pourra que les réjouir tant il rafraîchit la manière de livrer les ficelles du métier. Si Howard S. Becker (2002) s'en prenait aux difficultés des étudiants à mettre à distance leurs perceptions pour construire leur regard
* Sylvie Monchatre est maître de conférences en sociologie à l'université de Strasbourg. Elle a travaillé au Céreq pendant douze ans, où elle a développé des travaux sur le thème des usages sociaux de la compétence dans le travail et la formation. Elle a dernièrement publié : "Les carrières ouvrières à l'épreuve de la polyvalence. Une analyse de la situation française », avec Henri Eckert, dans la Revue Multidisciplinaire sur l'emploi, le syndicalisme et le travail (REMEST), vol. 4, n² 2, 2009, et " Normalisation des compétences et renouveau des politiques éducatives. Le cas de la formation technique au Québec», Recherches en Éducation, $n^{\circ} 7$, juin 2009. 
sociologique, Michel Arliaud s'en prend à leur tendance, presque opposée, à s'effacer derrière leurs observations. Trop souvent, dit-il en substance, la description vaut explication. Or, l'inventaire des faits, aussi rigoureux soit-il, n'a d'égal que son incapacité à rendre compte du social. Les chercheurs ne sont en aucun cas des "plaques photosensibles sur lesquelles le réel viendrait s'imprimer » (p. 181). Loin de toute vision positiviste, cet ouvrage appelle au contraire à l'audace du "pari interprétatif ». Il montre ainsi, avec audace, que la démarche scientifique tient finalement à peu de choses : elle relève de la présomption ainsi que d'hypothèses à mettre à l'épreuve sans preuves préalables. On mesure les risques encourus. Mais on ne saurait précisément les prendre sans cette "pulsion nécessaire à l'engagement dans la recherche ». Autrement dit, «si la part du désir que l'on a en nous de lever l'intrigue n'est pas forte, si l'on n'a pas cette espèce de curiosité, voire d'inquiétude fondamentale, je ne crois pas que l'on puisse bien vieillir dans ce métier » (p. 183).

Qui est celui qui parle ainsi ? Michel Arliaud est précisément un chercheur qui, parce qu'il a connu le doute, a tenu à transmettre les moyens d'y survivre. Cet ouvrage apporte un témoignage de son engagement pédagogique, d'autant plus précieux que Michel Arliaud est brutalement décédé en 2004. Spécialiste de sociologie de la médecine au sein du Laboratoire d'économie et de sociologie du travail (LEST) qu'il a dirigé pendant 10 ans, ancien directeur du centre associé du Céreq à Aix-en-Provence, il n’a eu de cesse d'entretenir et de faire partager avec gourmandise sa soif de sociologie. L'ouvrage a été réécrit à partir d'enregistrements des cours qu'il a donnés dans le cadre du DEA d'économie et de sociologie du travail entre 1990 et 2004. Et le message qu'il dispense à ses étudiants pourrait se résumer ainsi : osez le social ! Car le fait social n'existe que dans un rapport, il ne se donne pas à voir spontanément. Dès lors, tout l'enjeu de la démarche scientifique est d'en restituer la logique contre le sens commun qui le nie, mais aussi contre une certaine prétention scientiste à le saisir dans sa totalité et, enfin, contre l'idée qu'il n'y aurait qu'à le cueillir.

\section{POURQUOI EXPLICITER LE SOCIAL?}

L'enseignement de Michel Arliaud est une invitation à revenir aux auteurs fondamentaux, souvent délaissés dans le maelström de questions adressées aux experts du social. Comment, par exemple, faire face à une demande de recherche comparative entre médecine douce et médecine conventionnelle, sans tomber dans le piège d'avoir à cautionner une stratégie promotionnelle, en l'occurrence ici, des médecines douces (pp. 157-158)? Ce ne sont pas les propriétés intrinsèques de l'une et l'autre qui intéressent ici le sociologue, qui ne saurait déterminer le vrai du faux en la matière. Non : ici commence le pari interprétatif, il y a également une explication sociologique à leur surgissement. La demande des acteurs de la médecine parallèle ne peut, en l'occurrence, s'analyser que dans leur rapport aux médecines allopathiques, au sein d'un espace médical toujours plus éclaté en médecines de spécialités. Autrement dit, c'est à l'aide d'une pensée en termes de relations qui situe et pense un objet dans son espace relationnel que le regard sociologique va s'exercer.

Cette opération banale de requalification d'une demande sociale est le fruit de tout un héritage disciplinaire que Michel Arliaud s'emploie à retravailler et non à appliquer mécaniquement. Son enseignement ne manquait pas de rappeler (chap. 1) que la sociologie est précisément née de l'«impératif de justification des échanges humains » issu de la modernité : ce ne sont plus Dieu ou la nature qui justifient l'ordre du monde, ce sont les hommes qui s'en chargent et qui doivent s'en justifier. Mais la disparition de ces "garants métasociaux », selon l'expression de Touraine, ne signifie pas que toute détermination de l'ordre social a disparu, bien au contraire. La sociologie est née du projet d'expliquer en propre cette action de la société sur elle-même, ce qui suppose de rendre compte des déterminations qui orientent sa dynamique. Mais que d'obstacles à franchir dans cette entreprise ! 
Michel Arliaud a repéré les trois principaux obstacles qui sévissent, sur ce point, dans les sociétés occidentales. Le premier est celui du naturalisme : «Il y $a$ une espèce de génétique sauvage qui marque nos regards et nos propos sur le monde » (p. 41). De fait, nous cultivons la passion d'imputer à des propriétés individuelles les fonctionnements ou dysfonctionnements sociaux. C'est l'exemple de ce «directeur des ressources humaines qui va mettre en place un certain mode de gestion et, constatant que ça ne marche pas, que ces règles sont contournées par les salariés, en conclut : "ce sont des cons" "(Ibid). Pour Michel Arliaud, "c'est du naturalisme car les éléments de contexte qui déterminent les actions ne sont pas pris en compte " (Ibid). De fait, la sociologie s'oppose résolument à l'explication génétique et tout l'enjeu est de ne pas abdiquer prématurément le droit à l'explication sociologique.

Le deuxième obstacle est celui de l'égotisme (p. 43). L'idée que nous sommes socialement déterminés n'est jamais totalement acquise. Durkheim s'est précisément battu contre le primat de la conscience et de la volonté individuelle. Et la sociologie est née du refus des idéologies fonctionnelles qui placent l'individu (ego) « au principe de toutes les pratiques, des succès et des insuccès » (p. 44). Les historiens ont retenu la leçon avec l'école des Annales, qui suppose de renoncer à l'histoire des grands hommes. Mais force est de constater que la psychologie a irrigué le sens commun, dopée par l'idéologie méritocratique qui veut que le meilleur gagne sa position sociale. La fiction d'être "sans attaches et sans racines", comme disait Bourdieu à propos de la philosophie sartrienne du sujet libre, se diffuse d'ailleurs avec une radicalité surprenante, tant dans l'éducation, la pédagogie que dans le monde du travail et de l'emploi. Le «fantasme de l'homme auto-construit» (Rey, 2006) est plus fort que jamais, au point de nous faire oublier que la source de la liberté réside dans la confrontation aux déterminations sociales, davantage que dans leur déni. Et ces déterminations sont si lourdes qu'elles viennent se loger dans les profondeurs de la subjectivité, qu'on en juge par les quatre exemples donnés par Michel Arliaud (Chap. 3) : la réussite scolaire, le suicide, le coup de foudre, la maladie mentale.

\section{DE QUOI LE SOCIAL EST-IL LE NOM ?}

Le troisième obstacle à la démarche sociologique vient d'une conception de la science marquée par le positivisme : le réel y est conçu en extériorité, avec des lois propres, déchiffrables par la contemplation. Or, c'est à ce mythe de l'extériorité du sujet face au social (et inversement) que s'en prend Michel Arliaud. Son audace consiste ici à prendre ses distances, tout d'abord avec Durkheim lui-même, en reconstruisant la genèse de sa pensée. Durkheim a beaucoup insisté sur l'idée que le fait social suppose contrainte et extériorité, la sanction attestant de sa transgression. De fait, la seconde moitié du XIX ${ }^{e}$ siècle a été une période d'explosion des connaissances scientifiques et de foi en l'idée que l'on peut faire science de tout, qui lui imposait d'argumenter en écho à ce scientisme ambiant. Mais en réalité, l'extériorité du fait social est problématique tant la société s'incorpore en nous. La culture en est un bon exemple. Le système culturel que l'on fait sien est tellement fonctionnel qu'il ne saurait être sanctionné. Son caractère arbitraire (p. 95) tend à être oublié et même évacué dans l'idée d'un invariable déjà-là. Mais en réalité, il est le fruit d'une construction historique et s'objective dans des régularités statistiques. C'est donc ex post et non ex ante que la culture s'extériorise.

Mais le positivisme sévit à un autre endroit de l'histoire sociologique, en particulier dans la volonté de rendre compte du social dans un souci d'exhaustivité (Chap. 7). Gurvitch cherchait ainsi, à la suite de Mauss, à appréhender le "phénomène social total » comme un système démontable. Ses «paliers en profondeur » devaient permettre d'en explorer toutes les dimensions, de la «surface morphologique et écologique » de la société, aux "états mentaux » en passant par les " appareils organisés », etc. (p. 109). Or, une telle saisie de la totalité sociale présente non seulement une dimension normative, mais elle ne saurait prétendre à tout expliquer de la société, soumise à d'autres ordres de déterminations. Il importe donc, pour Michel Arliaud, de rester fidèle aux fondateurs dans leur ambition de penser le social comme une réalité sui generis non réductible à la somme des individus qui la composent, ainsi 
que d'expliquer le social par le social en tant que tel. Pour autant, il faut renoncer à l'illusion de la totalité pour se centrer sur les manifestations de la société dans les rapports sociaux : voilà le lieu de condensation du social.

Mais que sont les rapports sociaux ? On ne saurait les confondre avec des relations sociales, marquées par l'empirique, l'éphémère et le spécifique. Les rapports sociaux, au contraire, s’inscrivent dans la durée et présentent un haut degré de généralité : ils sont d'ordre théorique, ils donnent une lecture problématisée du social (p. 119). Ils ne sauraient, même s’ils les englobent indiscutablement, se réduire à des rapports de classe. C'est ainsi qu'un pari interprétatif établit l'existence de rapports sociaux entre les sexes, ainsi que le montre Anne-Marie Daune-Richard dans le chapitre 8. De fait, les groupes de sexe ne sont pas des «en-soi » séparés mais se définissent l'un par rapport à l'autre. Le rapport de domination qui les unit crée une structure sociale asymétrique et hiérarchisée, composée d'un pôle masculin dominant et constituant le référent général, et d'un pôle féminin, dominé, défini comme spécifique. Mais si les rapports sociaux ne font pas que traverser l'ensemble de la société, ils s'exercent également de manière spécifique à l'intérieur de champs sociaux, dans une configuration particulière.

Michel Arliaud emprunte à Pierre Bourdieu, avec qui il a longuement correspondu, cette notion de champ (chap. 9, p. 150) comme espace réel et théorique, à l'intérieur duquel se partage un même socle de croyance pratique ( « adhésion doxique »). Le champ se caractérise par deux propriétés structurales qui sont, d'une part, l'existence d'un capital spécifique et d'autre part, la distribution inégale de ce capital spécifique. Un champ n'est donc pas un long fleuve tranquille, il est traversé de tensions entre des "possédants » et des "prétendants", qui s'opposent dans le cadre de conflits de légitimité. C’est ainsi que le champ juridique a été secoué par le passage de la législation industrielle, calée sur un mode domestique de règlement des conflits de travail, au droit du travail (Cam, 1978). De même que le champ médical a connu un éclatement en «médecines de spécialités» dont le caractère « expéditif » s’est trouvé contesté par un corps de généralistes en quête de légitimité... et de spécialisation dans les médecines « lentes » et plus douces.
Par ailleurs, chaque champ possède une autonomie relative et ne saurait constituer l'unique horizon d'une vie. Célestin Bouglé (cité p. 78) met en évidence la pluralité des cercles sociaux d'appartenance des individus au sein d'une localité. Il en va de même avec les champs, qui nous font traverser un véritable «millefeuille social ». Faut-il en déduire, comme Bouglé, que nous sommes plus libres car nous n’y sommes pas enfermés? Le propos de Michel Arliaud se fait très nuancé à ce propos. Il souligne, à juste titre, qu'il n'y a pas de concordance entre les positions occupées dans différents champs : les dominants ne le sont pas toujours partout, il en va de même pour les dominés. Mais il peut en résulter des pratiques inattendues, conduisant moins à s'affranchir du poids de la domination ressentie qu'à en majorer les effets. Le fait d'être, ici en position dominante, là en position dominée, peut ainsi conduire à vivre cette situation comme stigmatisante et à en exhiber les effets (p. 178). Mais il peut également en résulter un travail de construction politique menant à des mobilisations inattendues : comment aurait pu avoir lieu, en 1968, l'alliance entre étudiants, culturellement dominants mais dominés dans le champ politique, et ouvriers, dominés sur les deux plans, sans travail d'énonciation de principes de rassemblement du type "dominés du pays, unissezvous!» (p. 170) ? Le principe «d'homologie structurale » mise en évidence par Bourdieu permet d'expliquer cette improbable alliance.

\section{LA RECHERCHE COMME POSTURE}

Cet ouvrage montre, enfin, que le social n'est jamais réductible à un lieu empirique juridiquement ou géographiquement délimité (une nation, une localité, une branche professionnelle, etc.). C'est aussi un «lieu théorique » (Chap. 5) que l'on ne peut voir qu'en se donnant les moyens de l'appréhender. Ce qui ne veut pas dire qu'il faut saisir des ordres de déterminations indépendamment des acteurs qui y contribuent. Pour Michel Arliaud, les sociologies de l'acteur seraient précisément recevables si elles n'oubliaient pas de rapporter les prises de position des «acteurs » aux conditions objectives qui ont 
permis leur émergence. De la même façon, le foisonnement d'études sur les identités gagnerait en clarté si, plutôt que de qualifier à l'infini les expériences de pluri-appartenance d'individus égarés, la sociologie s'employait à mieux rendre compte des dynamiques sociales qui, non seulement encadrent leur autonomie, mais également unifient leurs expériences a priori si hétérogènes.

Cette démarche suppose d'aller au-delà des discours tenus par les acteurs eux-mêmes sur leur propre situation et d'oser la mise en rapport social. C'est ici que resurgit l'audace du pari interprétatif, nécessaire pour suivre les « voies de la présomption ». Il faut, en effet, de l'audace pour collecter des données en faisant le pari qu'il en résultera des régularités, des corrélations auxquelles on ne donnera qu'ex post un sens sociologique. Mais de quel social cette audace à construire un objet sociologique en fin de recherche (et non au début) est-elle le nom ? Loin de se réduire à une qualité individuelle, elle renvoie, au contraire, à des conditions sociales de réalisation dont on peut se demander dans quelle mesure elles peuvent aujourd'hui continuer d'être réunies. On ne peut alors que remercier Michel Arliaud de nous inviter à ne pas y renoncer.

\section{Bibliographie}

Becker H.-S. (2002), Les Ficelles du métier. Comment conduire sa recherche en sciences sociales, Paris, La Découverte, Collection « Guide Repères ».

Cam P. (1978), « Juges rouges et droit du travail »,
Actes de la Recherche en Sciences sociales, 19, pp. 2-27.

Rey O. (2006), Une folle solitude. Le fantasme de l'homme auto-construit, Paris, Seuil.

\section{Référence de l'ouvrage}

Paul Bouffartigue, Jacques Bouteiller, Anne-Marie Daune-Richard, Henri Eckert et Annie Lamanthe, texte établi sous la responsabilité de Jacques
Bouteiller (2009), Pour une sociologie des rapports sociaux. L'enseignement de Michel Arliaud, Paris, La Dispute. 